Short note

\author{
L. Touati, T. Hamel \& A. Meddad-Hamza
}

\title{
Sur la présence d'Atriplex canescens (Amaranthaceae) en Algérie: écologie, taxonomie et biogéographie
}

\begin{abstract}
Touati, L., Hamel, T. \& Meddad-Hamza, A.: Sur la présence d'Atriplex canescens (Amaranthaceae) en Algérie: écologie, taxonomie et biogéographie. — Fl. Medit. 30: 33-38. 2020. - ISSN: 1120-4052 printed, 2240-4538 online.

On the occurrence of Atriplex canescens (Amaranthaceae) in Algeria: ecology, taxonomy and biogeography. - Atriplex canescens var. gigantea is recorded for the first time for the non-native flora of Algeria. The description of the finding locality, taxonomic notes and detailed distribution are provided.
\end{abstract}

Key words: Algeria, distribution, taxonomy, xenophytes.

\section{Introduction}

Dans le contexte mondial ou régional du bassin méditerranéen, les invasions biologiques constituent une menace permanente pour la biodiversité floristique avec la réduction de la flore spontanée (Lambdon \& al. 2008; Vila \& al. 2015).

En Algérie, les invasions végétales ont été peu étudiées (Ducellier \& Maire 1923; Dobignard \& Chatelain 2010-2013; Kazi Tani \& al. 2012; Véla \& al. 2013; Kazi Tani 2014; Meddour \& El Mokni 2016; Hamel 2016; Adjim \& Kazi Tani 2018; Miara \& al. 2018; Hamel \& Azzouz 2018; Sakraoui \& al. 2019). La synthèse bibliographique réalisée par Meddour \& El Mokni (2016) a montré que 45,29 \% des plantes introduites en Algérie sont des plantes naturalisées, dont $18 \%$ sont considérées comme envahissantes ou potentiellement envahissantes.

La composition taxonomique de la flore exotique n'est pas aléatoire et les familles avec le plus grand nombre de plantes xénophytes appartiennent aux plus grandes familles indigènes du monde (Pyšek, 1998; Pyšek \& al. 2002, 2017). En effet, cette flore est dominée par les familles, Asteraceae Martivon et des Amaranthaceae Juss. sensu APG IV (2016).

Le genre Atriplex L. contient environ 260 espèces dont la majorité sont distribuées dans les régions arides et semi-arides d'Eurasie, d'Amérique et d'Australie (Sukhorukov \& Danin 2009; Kadereit \& al. 2010; Brignone \& al. 2016). L'Algérie est représentée par 17 taxons dont deux sont naturalisées et un est adventice. 
L'Atriplex canescens (Pursh) Nutt. est naturalisé en Egypte, Tunisie et Maroc (Dobignard \& Chatelain 2011; Iamonico \& El Mokni 2019). Son existence en Algérie n'a jamais été signalée. Nous rapportons ici pour la première fois la présence de cette espèce en tant que plante étrangère naturalisée de la flore algérienne.

\section{Contexte de la découverte}

En septembre 2018, lors de prospections botaniques habituelles entretenues dans les zones limitrophes des formations préforestières et steppiques du Tell et hauts plateaux Constantinois $\left(\mathrm{C}_{1} \& \mathrm{H}_{2}\right.$ selon la subdivision biogéographique proposée par Quézel \& Santa 1962), zones connues pour leur richesse floristique, l'un des auteurs (H.T) a récolté un spécimen d'Atriplex, dans les grandes steppes à Macrochloa tenacissima (L.) Kunth et Artemisia herba-alba Asso de Ben Attia (Souk Ahras Nord-Est algérien) (Fig. 1). L'examen de la plante a montré qu'il s'agit d'A. canescens var. gigantea, une nouvelle plante pour la flore algérienne (cf. Battandier 1888-1890; Battandier \& Trabut 1905; Maire 1952-1987; Quézel \& Santa 1962-1963; Dobignard \& Chatelain 2011). Une deuxième visite en décembre 2019, nous a permis de recenser une vingtaine de pieds de cette plante avec une végétation xérophile/ halophile: Argyrolobium saharae Pomel, Atractylis caespitosa Desf., Atriplex halimus L., Centaurea pubescens Willd., Deverra scoparia Coss. \& Durieu, Ebenus pinnata Aiton, Ephedra alata subsp. alenda (Stapf) Trab., Ephedra major Host, Globularia alypum L., Olea europaea L. subsp. europaea, Ononis angustissima subsp. polyclada Murb., Opuntia ficus-indica (L.) Mill., Peganum harmala L., Limonium echioides (L.) Mill., Lotus halophilus Boiss. \& Spruner, Lygeum spartum L., Mandragora officinarum L., Marrubium alysson L., Santolina africana Jord. \& Fourr., Sedum sediforme (Jacq.) Pau., Sideritis incana L., Tamarix gallica L., Teucrium capitatum L. et Thymus algeriensis Boiss. \& Reut.

Deux spécimens collectés ont été déposés dans l'herbier du département de Biologie, faculté des Sciences, université Badji Mokhtar Annaba-Algérie.

\section{Description taxonomique et répartition géographique}

Atriplex canescens (Pursh) Nutt [ $\equiv$ Calligonum canescens Pursh; $\equiv$ Obione canescens (Pursh) Moq.; 三 Pterochiton canescens (Pursh) Nutt.].

Arbuste dioïque de 10 a $18 \mathrm{dm}$ de long, avec une tige dressée, très ramifiée et pubescente. Les feuilles sont alternes linéaires-oblongues, subsessiles à courtement pétiolées, mucorinées à l'apex et a rùrvêtement entièrement blanchâtre (Fig. 2). L'inflorescence de la plante est en panicules spiciformes, elle est fixée sur l'extremite des rameaux fertiles avec de nombreux glomérules (Flore du Maroc 2019).

Atriplex canescens est une espèce néophyte originaire d'Amérique du Nord et du Mexique, alors qu'elle est étrangère en Australie (Atlas of Living Australia 2016), en Asie orientale (Freitag \& al. 2001), en région de la Méditerranée orientale (Boulos 1999) et en Afrique (SANBI 2012). Elle est rencontrée dans les écosystèmes arides et désertiques du monde, où elle constitue une source fourragère importante pour le betail (El Shaer 2010; Mellado \& al. 2012). 
Atriplex canescens c'est une espèce assez variable. Taxonomiquement, cette variabilité a été distinguée au niveau variétal. En plus de la variété nominale (A. canescens var. canescens), le Plants Database du USDA/Natural Resources Conservation Service (2020) signale les cinq variétés suivantes:

- A. canescens var. angustifolia (Torr.) S. Watson;

- A. canescens var. gigantea S.L. Welsh \& Stuz;

- A. canescens var. laciniata Parish;

- A. canescens var. linearis (S. Watson) Munz;

- A. canescens var. macilenta Jeps.

Les pieds d'Atriplex canescens trouvés en Algérie correspond à la var. gigantea.

L'âge des pieds observés de la plante en question avoisine les 15 à 20 ans. En se basant sur la littérature et nos prospections périodiques dans cette zone steppique, il est fort probable qu'au cours de ces dernières années cette espèce a été naturalisée pour réhabiliter des parcours en zones dégradées en raison de sa plus grande résistance au froid (Forti 1986). Ses caractéristiques écologiques et physiologiques spécifiques lui permettent de se développer vigoureusement dans les parcours steppiques (Henni \& Mehdadi 2011). Bien enten$\mathrm{du}$, la naturalisation des espèces xénophytes sur le territoire algérien est un processus continu, car de nombreuses espèces ont été déjà signalées (Véla \& al. 2013).

L'utilisation des plantations pastorales à Atriplex canescens var. gigantea, par le Haut Commissariat au Développement de la Steppe (HCDS), constitue une approche prometteuse dans la lutte contre la désertification. Il présente une tolérance à la salinité, la sécheresse

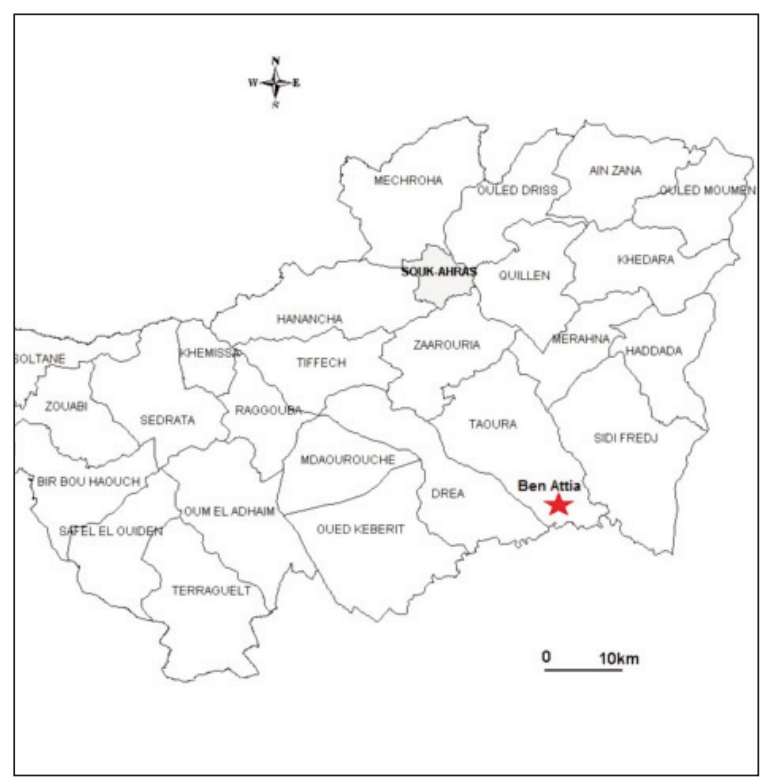

Fig.1. Localisation de la station d'observation.

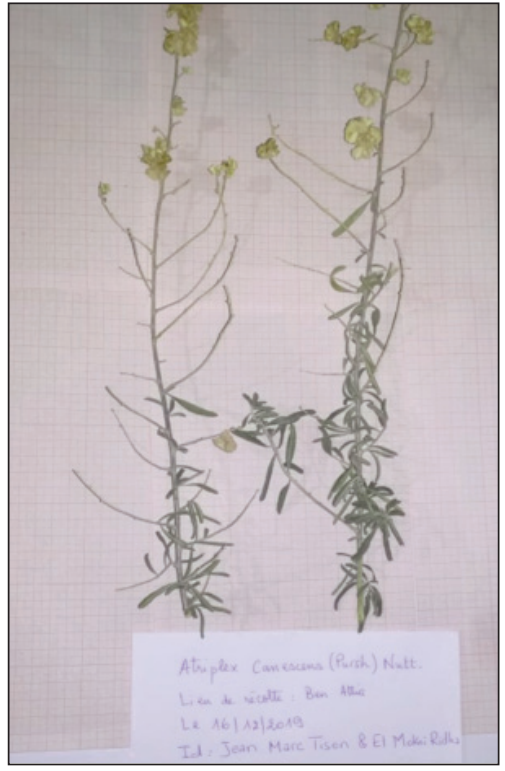

Fig. 2. Le spécimen prélève (Clichés de Leila TOUATI). 
et aux métaux lourds (Shabala 2013). Ainsi, il possède un système racinaire très développé fixant les couches supérieures du sol et constitue un matériel biologique de choix pour l'enrichissement de la flore et la protection du sol dans les zones arides et semi-arides (Belkhodja \& Bidai 2004; Le Houérou 2006).

Plusieurs pieds de la plante sont observés aux milieux des champs de cultures de la région de Ben Attia. Ce comportement est un bon indicateur de son caractère et son potentiel invasif (Brunel \& Tison 2005).

\section{Conclusion}

La découverte d'Atriplex canescens var. gigantea dans les steppes de Souk Ahras ajoute une nouvelle espèce naturalisée pour la flore algérienne et elle constitue un ajout important pour la flore fourragère du pays.

Ce taxon a été planté pour la réhabilitation des parcours dégradés des zones steppiques algériennes. Donc, il est fort possible que d'autres populations de la plante sont présentes sur le territoire national.

\section{Remerciements}

Nous tenons à remercier M. Jean-Marc Tison et M. El Mokni Ridha pour leurs aides dans l'identification taxonomique de la plante. Nous remercions M. Alalga Mounir pour sa disponibilité et sa présence au cours de la prospection du terrain.

\section{Références}

Adjim, Z. \& Kazi Tani, C. 2018: L'infestation par Solanum elaeagnifolium menace l'Algérie. - Rev. Ecol. (Terre Vie) 73(4): 569-581.

APG IV 2016: An update of the Angiosperm Phylogeny Group classification for the orders and families of flowering plants: APG IV. - Bot. J. Linn. Soc. 181: 1-20. https://doi.org/10.1111/boj.12385

Atlas of living Australia 2016+: Atriplex canescens (Pursh) Nutt. http:// bie.ala.org.au/species/http://id.biodiversity.org.au/node/apni/2910967 [Dernier accès 10/02/2020]

Battandier, J. A. 1888-1890: Flore d'Algérie: Ancienne flore d'Alger transformée (Dicotylédones). - Alger.

— \& Trabut, L. C. 1905: Flore analytique et synoptique de l'Algérie et de la Tunisie. - Alger.

Belkhodja, M. \& Bidai, Y. 2004: Réponse des graines d'Atriplex halimus L. à la salinité au stade de la germination. - Sécheresse 15: 331-335.

Brunel, S. \& Tison, J.M. 2005: A method of selection and hierarchization of the invasive and potentially invasive plants in continental Mediterranean. France. - Pp. 34-43 in: Brunel, S. (ed.), Plantes envahissantes dans les régions méditerranéennes du monde. - Mèze.

Boulos, L. 1999: Flora of Egypt, 1. - Cairo.

Brignone, N. F., Denham, S. S. \& Pozner, R. 2016: Synopsis of the genus Atriplex (Amaranthaceae, Chenopodioideae) for South America. - Australian Syst. Bot. 29: 324-357. http://doi.org/10.1071/ SB16026 
Dobignard, A. \& Chatelain, C. 2010-2013: Index synonymique et bibliographique de la Flore d'Afrique du Nord, 1-5. - Genève.

Ducellier, L. \& Maire, R. 1923: Végétaux adventices observés dans l'Afrique du Nord. -Bull. Soc. Hist. Nat. Afrique N. 14: 304-325.

El Shaer, H. M. 2010: Halophytes and salt-tolerant plants as potential forage for ruminants in the Near East region. - Small Rumin. Res. 91: 3-12. https://doi.org/10.1016/j. smallrumres. 2010.01 .010

Flore du Maroc 2019: http://www.floramaroccana.fr/presentation.html. [Dernière consultation 10/02/2020].

Forti, M. 1986: Salt-tolerant and halophytic plants in Israel. - Recl. Reveg. Res. 5(1-3): 38-96.

Freitag, H., Hedge, I. C., Jafri, S. M. H., Kothe-Heinrich, G., Omer, S. \& Uotila, P. 2001: Atriplex L. - Pp. 54-70 in: Ali, S. I \& Qaiser, M. (eds): Flora of Pakistan, 204. - St. Louis.

Hamel, T. 2016: Première observation d'une xérophyte Oenothera rosea L'her. ex Aiton. (Onagraceae) en Afrique du Nord. - Acta Bot. Malacitana 41: 287-289. https://doi.org/10.24310/abm.v41i0.2460

— \& Azzouz, Z. 2018: Découverte de Gamochaeta antillana (Asteraceae) en Numidie orientale (El Tarf-Algérie). - Fl. Medit. 28: 155-164. doi: 10.7320/FlMedit28.155

Henni, M. \& Mehdadi, Z. 2012: Évaluation préliminaire des caractéristiques édaphiques et floristiques des steppes à armoise blanche dégradées réhabilitées par la plantation d'Atriplex dans la région de Saïda (Algérie occidentale). - Acta Bot. Gallica 159: 43-52. https://doi.org/10.1080/12538078.2012.671640

Iamonico, D. \& El Mokni, R. 2019: On Atriplex canescens (Chenopodiaceae s. str./ Amaranthaceae s. 1.) in Tunisia: nomenclatural and morphological notes on its infraspecific variability. Haquetia 18(1): 119-127. http://doi.org/10.2478/hacq-2018-0008

Kadereit, G., Mavrodiev, E.V., Zacharias, E.H. \& Sukhorukov, A. P. 2010: Molecular phylogeny of Atripliceae (Chenopodiaceae): Implications for systematics, biogeography, flower and fruit evolution, and the origin of $\mathrm{C}_{4}$ photosynthesis. - Amer. J. Bot. 97(10): 1664-1687. http://doi.org/10.3732/ajb.1000169

Kazi Tani, C., Le Bourgeois, T \& Munoz, F. 2012: Alien versus native weeds present in crops of Oranie (North West Algeria): A comparative study of their life-history traits. - Fl. Medit. 22: 33-44. http://doi.org/10.7320/FlMedit22.033

- 2014: Biologie et écologie d'une nouvelle plante parasite en Algérie: Cuscuta campestris Yunck. (Convolvulaceae). - Poiretia 6: 1-15.

Lambdon, P. W, Pyšek, P., Basnou, C., Hejda, M., Arianoutsou, M., Essl, F., Jarošíc, V., Pergl, J., Winter, M., Anastasiu, P., Andriopoulos, P., Bazos, I., Brundu, G., Celesti-Grapow, L., Chassot, P., Delipetrou, P., Josefsson, M., Kark, S., Klotz, S., Kokkoris, Y., Kühn, I., Marchante, H., Perglová, I., Pino, J., Vilà, M., Zikos, A., Roy, D. \& Hulme, P.E. 2008: Alien flora of Europe: species diversity, temporal trends, geographical patterns and research needs. - Preslia 80: 101-149. https://doi.org/10.17011/conference/eccb2018/107390

Le Houérou, H. N. 2006: Agroforestry and sylvopastoralism: The role of trees and shrubs (Trubs) in range rehabilitation and development. - Sécheresse 17: 343-348.

Maire, R. 1952-1987: Flore de l'Afrique du Nord (Maroc, Algérie, Tunisie, Tripolitaine, Cyrénaïque et, Sahara), 1-16. - Paris.

Meddour, R. \& El Mokni, R. 2016: Etat de l'art sur les plantes envahissantes ou à caractère invasif introduites en Algérie et en Tunisie. - P. 100 in: Médail, F. \& Domina, G. (eds), XV OPTIMA Meeting, Abstracts. - Montpellier.

Mellado, M., Rodríguez, A., Lozano, E. A., Dueñez, J., Aguilar, C. N. \& Arévalo, J. R. 2012: The food habits of goats on rangelands with different amounts of fourwing saltbush (Atriplex canescens) cover. - J. Arid Environ. 84: 91-96. https://doi. org/10.1016/j.jaridenv.2012.03.012 
Miara, M. D., Boutabia, L., Telaïlia, S. \& Véla, E. 2018: Apparition de Senecio angulatus (Asteraceae) en Algérie. - Fl. Medit. 28: 111-118. https://dx.doi.org/10.7320/FlMedit28.111

Pyšek, P. 1998. Is there a taxonomic pattern to plant invasions? - Oikos 82: 282-294. . https://doi. org $/ 10.2307 / 3546968$

—, Richardson, D. M., Rejmanek, M., Webster, G. L., Williamson, M. \& Kirschner, J. 2002: 'Alien plants in checklists and floras: towards better communication between taxonomists and ecologists'. - Taxon 53: 131-143. http://dx.doi.org/10.2307/4135498

—, Pergl, J., Essl, F., Lenzner \& al. 2017. Naturalized alien flora of the world: species diversity, taxonomic and phylogenetic patterns, geographic distribution and global hotspots of plant invasion. - Preslia 89(3): 203-274. https://dx.doi.org/10.23855/preslia.2017.203

Quézel, P. \& Santa, S. 1962-1963: Nouvelle flore de l'Algérie et des régions désertiques méridionales, 1-2. - Paris.

Sakhraoui, N, Metallaoui, S., Chefrour, A. \& Hadef, A. 2019: La flore exotique potentiellement envahissante d'Algérie: première description des espèces cultivées en pépinières et dans les jardins. - Biotechnol. Agron. Soc. Environ. 23(2): 63-73.

SANBI 2012: Atriplex canescens (Pursh) Nutt. - In: National Assessment: Red List of South African Plants version 2014.1. - http://redlist.sanbi.org [Dernier accès 1/2/2020]

Shabala, S. 2013: Learning from halophytes: Physiological basis and strategies to improve abiotic stress tolerance in crops. - Ann. Bot. 112: 1209-1221. http://doi.org/10.1093/aob/mct205

Sukhorukov, A.P. \& Danin, A. 2009: Taxonomic notes on Atriplex sect. Teutliopsis and sect. Atriplex in Israel and Syria. - Fl. Medit. 19: 15-23.

USDA/Natural Resources Conservation Services 2020: Plants Database https://plants.usda.gav.core/profile?simbol=ATCA2 [Dernière consultation 10/02/2020].

Véla, E., Rebbas, K., Meddour, R. \& de Bélair, G. 2013: Notes et compléments sur quelques taxons traités dans les volumes 1 à 4 . Note sur quelques xénophytes nouveaux pour l'Algérie (et la Tunisie). - Pp. 372-376 in: Dobignard, A. \& Chatelain, C. (eds) Index synonymique Flore d'Afrique du Nord, 5. - Genève.

Vilà, M., Rohr R. P., Espinar J. L., Hulme P. E., Pergl J., Le Roux, J. J. \& Pyšek, P. 2015: Explaining the variation in impacts of non-native plants on local-scale species richness: the role of phylogenetic relatedness. - Glob. Ecol. Biogeogr. 24: 139-146. https://doi.org/10.1111/geb.12249

Adresse des auteurs:

Leila Touati, Tarek Hamel* \& Amel Meddad-Hamza,

Département de Biologie, Faculté des Sciences, Université Badji Mokhtar-Annaba, Algérie. BP. 23000. E-mail: tarek_hamel@yahoo.fr

*Auteur correspondant. 